\title{
Cancer Vaccines: Bench to Bedside
}

\author{
Neha Tuli ${ }^{\dagger, 1}$, Rachana Maniyar ${ }^{\dagger, 1}$, Robert Bednarczyk ${ }^{1}$, \\ Ghada Ben Rahoma ${ }^{1}$, Sarnath Singh ${ }^{1}$, Jan Geliebter ${ }^{1}$, \\ Abraham Mittelman $^{1}$, Marc Wallack ${ }^{1,2}$, Debabrata Banerjee ${ }^{3}$ \\ and Raj K. Tiwari ${ }^{1, *}$ \\ ${ }^{1}$ Departmentof Microbiology and Immunology, New York Medical College, \\ Valhalla, NY 10595, USA \\ ${ }^{2}$ Department of Surgery, Metropolitan Hospital Centre 1901 1st Avenue, \\ New York, NY 10029, USA \\ ${ }^{3}$ Department of Pharmacology, Rutgers, The State University of New Jersey, \\ Piscataway, NJ 08854, USA \\ $\dagger$ These authors contributed equally to the preparation of this manuscript \\ *Corresponding Author: Raj_tiwari@nymc.edu
}

Received 2 March 2016; Accepted 16 March 2016;

Publication 25 April 2016

\begin{abstract}
The immune system has immense potential in cancer therapy as it is individualized, precision driven and robust, however, it is associated with challenges of its own that include immune evasion, development of tolerance and a sustained tumor rejection response. Recent FDA approval of several checkpoint inhibitors, anti-CTLA4, anti-PD-1, has re-invigorated cancer immunology by demonstrating that tolerance to cancer can be broken to induce a sustained immune response in patients. Active immunization with multivalent tumor associated antigens (TAA), however, is still a challenge. We have developed two specific distinct methods to generate multivalent antigens capable of tumor regression in prostate cancer and melanoma. In prostate cancer, we have generated specific multivalent peptide mimetics using phage display synthetic peptide libraries capable of metastatic tumor regression in an animal model. In melanoma, we have used a vaccinia virus based antigen retrieval technology to generate a multivalent antigenic vaccine. The antigenic repertoire is well defined. A protocol for the melanoma vaccine is FDA approved for clinical
\end{abstract}

International Journal of Translational Science, Vol. 2016, 33-60.

doi: 10.13052/ijts2246-8765.2016.003

(c) 2016 River Publishers. All rights reserved. 
trials. We envision defining the humoral and cellular immune response to combine our active vaccine strategy with other treatment modalities including approved checkpoint inhibitors anti-CTLA4 and anti-PD-1. We believe our vaccine candidates are a new generation of immune-therapeutics that can prolong cancer free survival and prevent secondary recurrences. Our studies have challenged the existing paradigms to re-define cancer immunotherapy that bridges the gap between humoral and cellular immunity by combining active immune response with negative checkpoint inhibitors thus activating pre-existing dormant immune response.

Keywords: cancer vaccines, peptide epitopes, vaccinia virus - antigen retrieval.

\section{Introduction}

Cancer immunotherapy has come of age and is now on the verge of becoming a mainstream therapy. The work done by Baldwin, Foley, Prehn, Main and Klein [1-4] using chemically induced tumor immunogenicity analyses have enabled the identification of tumor associated antigenic epitopes. Many of these identified epitopes found their way into a clinical setting. The clinical success was, however, limited due to the lack of our ability to launch and sustain a robust immune response capable of eliminating cancers. Utilization of the role of both arms of the immune system led to B cell and T cell defined antigenic epitopes densely populating the landscape of specific cancers, warranting testing against the clinical yardstick. The lack of clinically responsive results using these cancer associated antigenic epitopes brought us back to the drawing board, although with some significant lessons. These lessons form the basis of present successes; however, there remains the need for further refinement to successfully launch mainstream cancer immunotherapy. These studies reveal that cancers are immunogenic, having multivalent epitopes and a reasonable clinical success should target the complete repertoire of cancer epitopes. Thus, we need to develop a method by which we can sustain an immune response and ultimate elimination of tumors should target a positive stimulatory pathway of both $\mathrm{B}$ and $\mathrm{T}$ cell defined antigenic epitopes and release the tumor of its negative regulatory effects by overcoming the tumor induced immunosuppressive environment. Our understanding of the multivalent nature of cancer resulting from genomic instability forms the backbone of tailor-made individualized precision medicine which seems to be the marching order of present times. 
The generation of tumor associated antigenic epitopes is an orchestrated activity, initiated by abnormal translational, post-translational, proteosomal processing and/or genetic instability [5, 6]. Tumor specific antigens (TSAs) represent neoantigens that arise from somatic mutations or oncogenic viral proteins. Somatic mutations can either be driver mutations, causing transformation, or genomic instability leading to passenger mutations. These mutations are recognized by the host immune system as foreign, resulting in an adaptive immune response in experimental animals and cancer bearing hosts. It is assumed that neoantigen specific cytotoxic $\mathrm{T}$ cell responses against HLA matched tumor cells may form the basis of successful future cancer immunotherapies [7, 8].

\subsection{Peptide Epitopes}

Previous mice studies suggest existence of 'specificity' in cancer immunity $[1-4,9]$. Study of mice immunized against syngeneic carcinogen induced tumors, indicated that a commonly expressed protein can be used to immunize these mice against cancer. These molecules were later identified to be heat shock proteins 70 and 90, both chaperone proteins [10]. These heat shock proteins when purified from tumor homogenates, mediated tumor rejection in animal studies. Hence they were also known as "tumor rejection antigens" or TRAs. However, later studies observed the immunogenicity to be due to short peptides that were infact noncovalently linked with these HSPs and acting as immunogens. HSP receptors, present on dendritic cells, become a point of interaction for HSPs associated with the immunogens. Thus, HSPs only assist in delivering these immunogenic peptides to dendritic cells which in turn can trigger a cytotoxic $\mathrm{T}$ cell response against the cancer cells $[11,12]$. This proved that HSP actually played an indirect role in immunogenicity rather than a direct one. Hence secreted HSPs from cancer cells have immuneenhancing roles. The action of HSPs depends on their innate characteristics; for example whether they are secreted in bound form or within exosomes, rate of secretion and the subset of HSP secreted (hsp70, gp96, hsp90, hsp110, etc). Srivastava and colleagues have shown that hsp70 and gp96 can bind to tumor antigenic polypeptides forming complexes. These HSP-peptide complexes can be utilized for generation of an anti-cancer vaccine [10, 13-15] and have been a major source of multivalent epitopes. We embarked upon a program of generating peptide mimotopes using a combinatorial single chain antibody library to identify and isolate synthetic peptides that mimicked the 
immunological tumor rejection properties of HSPs. Some of these study results have been described below.

\subsection{Multivalent Therapeutic Vaccines: The Need of the Hour}

Multiple successes in using peptide based vaccines for infectious diseases indicate that peptides, being highly immunogenic, are prime candidates to mount a vaccine driven antigen specific immune response. Genomic instability, particularly erroneous end joining and translocations, play a primary role in causing malignancies expressing neoantigens [16]. Trials using synthetic peptides coupled with Incomplete Freund's Adjuvant (IFA) have shown some promise in terms of inducing antigen specific $T$ cell responses. However, due to the high heterogeneity in mutation and expression profiles of these neoantigens on tumor cells, trials using single antigenic vaccines have shown to induce an antigen specific immune response, albeit inadequate in the clinic [17].

We have pursued the concept of using multivalent cancer antigenic epitopes by two distinct technologies; the first being a peptide mimotope technology and the second a vaccinia virus based antigen retrieval system. The peptide mimotopes were generated against cancer specific HSPs with the rationale that these HSPs by virtue of their peptide chaperoning property would harbor tumor associated antigens and that a true immunological mimic would emulate the tumor rejection properties of these HSPs; inducing a sustained, tumor specific and robust cytotoxic $\mathrm{T}$ cell response (CTL) to eliminate tumors. Further these synthetic peptides with little or no resemblance to endogenous protein sequences, generate a significantly active antibody response. Development of this immunogen is the first of its kind, expected to stimulate both arms of the immune system with a single focused aim of tumor regression.

We also utilized an alternative approach, whereby, we isolated and characterized five patient derived metastatic primary melanoma cell lines. We infected these cell lines with vaccinia virus and used the membrane oncolysates as a vaccine. The rationale being that vaccinia virus in the process of budding out of the membrane will be associated with the entire repertoire of melanoma associated antigens (MAA) which can be used as a ready source of multivalent antigens. This repertoire consists of tumor specific and pan antigens together with its natural adjuvant, the vaccinia virus $[18,19]$. In this communication we provide an outline of the success of both these approaches and suggest a clinically relevant additional combinational use of immunogens. 


\section{Results}

\subsection{Multivalent Cancer Vaccine Using HSP Peptide Mimotopes as Immunogens}

The general strategy of the generation of peptide mimotopes is initiated by differential panning of a single chain antibody library with tumor as well as normal tissue derived HSP; in this case hsp96. This strategy through a rigorous amplification procedure gives rise to HSP tumor specific single chain antibodies which are purified, and used as reagents in subsequent panning of combinatorial synthetic peptide libraries to give rise to peptide mimotopes. One such typical experimental result using MAT-LyLu rat cell line derived HSPs are depicted in Figure 1 and Figure 2. The experiment showed that not only can tumor specific hsp96, a CTL inducer, be recognized by single chain antibodies, but also that synthetic peptides (mimotopes) not represented in the proteome, can be identified. The sequence specificity of these peptides (Table 1) is a testimony that these mimotopes derived are not library specific and indeed both linear and conformational epitopes can be generated. We have used these peptides to predict an HLA binding score (Table 2) and further defined their efficacy as an immunotherapaeutic vaccine. Prior vaccination of the animals and subsequent challenge with the tumor completely abrogated tumor growth (Figure 3).

\subsection{Multivalent Cancer Vaccine Using Vaccinia Based Antigen Retrieval System}

The use of vaccinia virus based antigen retrieval system was designed to be tested using human melanoma primary cells that were isolated from patients with metastatic lesions. Procedural details for cell isolation, growth and infection with vaccinia, preparation of the oncolysate have been described elsewhere [19-21]. The rationale of such a scheme was entrenched in basic viral processes of budding whereby the virus always 'pinches' membrane proteins. We used the membrane preparation prior to the budding of the virus and the use of five different primary cells reassured us of a combined preparation that was endowed with a vast repertoire of multivalent MAA. Additionally, in vitro studies conducted by our group have shown an enhanced expression of MAAs on treatment of these cell lines with vemurafenib, a BRAFV600E inhibitor, warranting its possible use in enhancing our oncolysate vaccine efficacy $[22,23]$. 


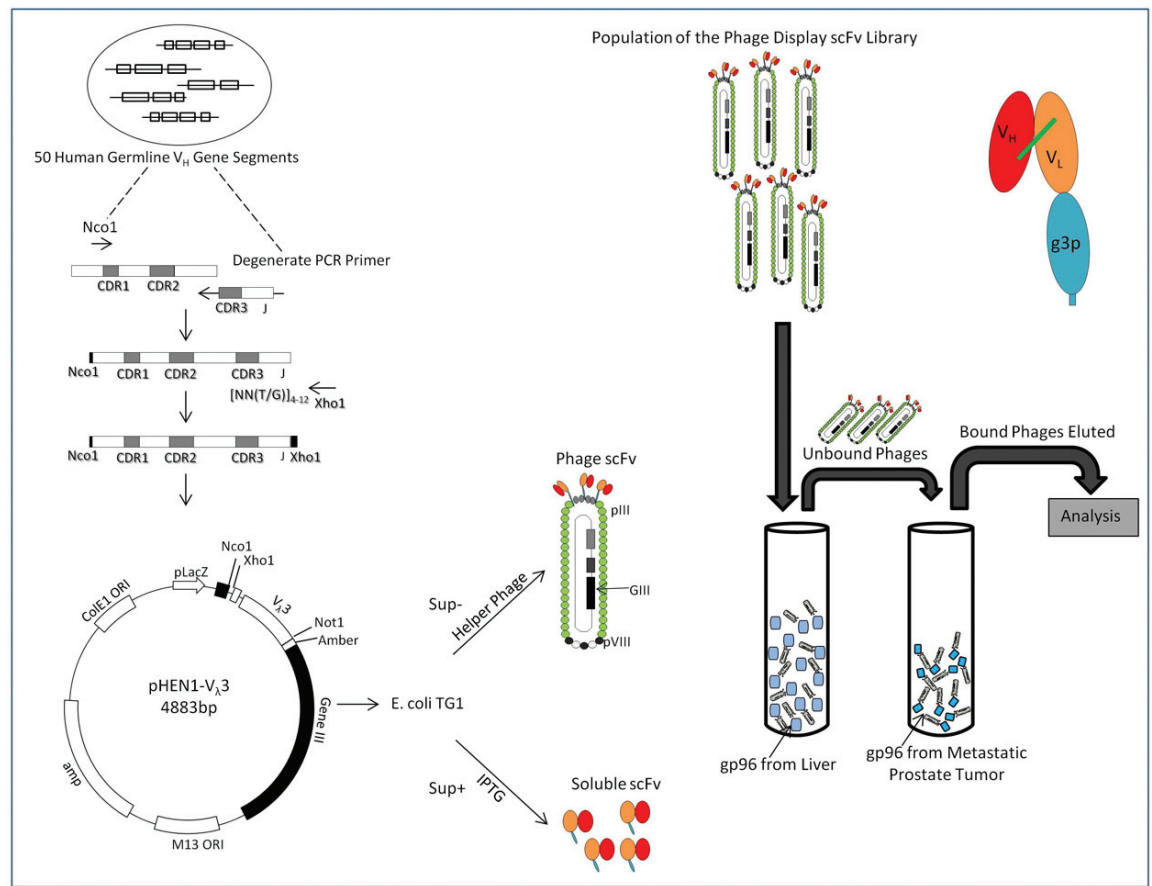

Figure 1 Phage Display Library and Panning Strategy for generation of single chain antibodies specific to hsp96: Randomized CDR3 regions were inserted into multiple cloning sites of phagemid and fused with gene III of bacteriophage M13 capsid protein. The phage was inserted into $\mathrm{E}$ coli, a gram negative bacteria, for amplification to generate a single chain antibody ( $\mathrm{scFv}$ ) phage library. Hsp96 was panned against this library. scFv phage with affinity and specificity to hsp96-peptide complex was selected using proteins adsorbed to Maxisorp immunotubes. Four rounds of panning carried out using immunotubes coated with hsp96 complex overnight at varying concentration of $10 \mu \mathrm{g} / \mathrm{ml}, 5 \mu \mathrm{g} / \mathrm{ml}$ and two rounds of $2.5 \mu \mathrm{g} / \mathrm{ml}$ in PBS for first, second, third and fourth rounds respectively. After a 2 hour incubation of scFv phages with the hsp96 coated tubes, unbound/ non-specifically bound scFvs were eluted with PBS/PBST washes. The hsp96-peptide complex bound phages were eluted with elution buffer and put through further rounds of selection for metastatic prostate tumor (MAT-LyLu) specific hsp96 complex. The tumor specific scFv phage clones were then immunoscreened and analyzed [56, 57].

In a compassionate human clinical trial (Figure 4) we used autologous DCs pulsed with oncolysates derived from primary melanoma cell lines infected with recombinant IL-2 gene encoded vaccinia virus to define the immunological parameters that are shown in Table 3 . We observed partial and complete responses that were augmented by the use of IL-2 prior to vaccination, again a reminder of the need to emulate the natural environment of a tissue specific immune mediated inflammatory response. 


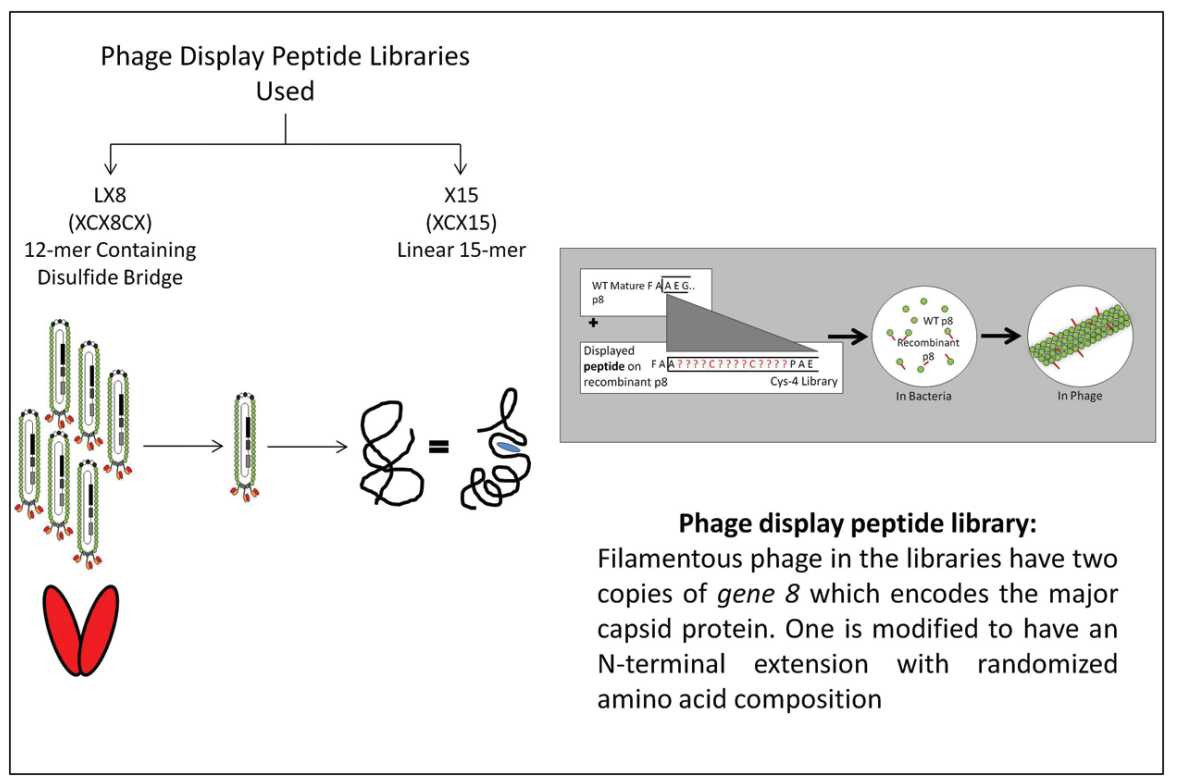

Figure 2 Identification of peptide mimotopes to metastatic tumor derived hsp96 from a phage display combinatorial peptide library: The E6 clone, a MAT-LyLu specific scFv, was panned against two phage display peptide libraries LX8 (12 mer containing disulphide bridge) and X15 (linear 15-mer).

Table 1 Amino acid sequence of synthetic peptides derived by panning with scFv E6: LX8 (12-mer containing disulphide bridge) and X15 (linear 15-mer mimotope of MAT-LyLu gp96peptide complex)

\begin{tabular}{|c|c|}
\hline Library & Sequence \\
\hline LX-8 & YCQEGDSPRLCL \\
X-15 & $\begin{array}{c}\text { YQPPSDALRWILRLQ } \\
\text { GQWOSGDRYWMETST }\end{array}$ \\
\hline
\end{tabular}

\section{Discussion}

It is known that during the education of $\mathrm{T}$ lymphocytes in the thymus, $\mathrm{T}$ cells are MHC restricted i.e. they only recognize self MHC complexed with peptides. Additionally, not all peptides bind to all MHC molecules; the binding is determined by the size and the charge of the peptide, thus adding one more level of restriction [24]. Flexibility conferred by peptide based vaccines, 
Table 2 Peptide match to HLA Class I: HLA binding score predicted for the two peptides $\mathrm{X}-15$ and LX-8b

\begin{tabular}{|c|c|c|c|c|c|}
\hline \multicolumn{3}{|c|}{$\begin{array}{c}\text { BTE6-X-15-7 } \\
\text { (GQWQSGDRYWMETST) }\end{array}$} & \multicolumn{3}{|c|}{$\begin{array}{c}\text { BTE6-LX-8b } \\
\text { (YCQEGDSPRLCL) }\end{array}$} \\
\hline & HLA Allele & Binding score & & HLA Allele & Binding score \\
\hline 1. & HLA-A-01 & $\begin{array}{l}17 \\
12\end{array}$ & 1. & HLA-A-01 & 14 \\
\hline 2. & HLA-A-0201 & 9 & 2. & HLA-A-0201 & 12 \\
\hline 3. & HLA-A-03 & 9 & 3. & HLA-A-03 & 10 \\
\hline 4. & HLA-A-26 & 11 & 4. & HLA-A-2402 & $\begin{array}{l}10 \\
10\end{array}$ \\
\hline 5. & HLA-B-0702 & 11 & 5. & HLA-A-26 & 20 \\
\hline 6. & HLA-B-1510 & 9 & 6. & HLA-B-0702 & $\begin{array}{l}18 \\
13\end{array}$ \\
\hline 7. & HLA-B-2705 & $\begin{array}{l}16 \\
15 \\
11\end{array}$ & 7. & HLA-B-08 & $\begin{array}{l}12 \\
10\end{array}$ \\
\hline 8. & HLA-B-2709 & $\begin{array}{l}12 \\
10\end{array}$ & 8. & HLA-B-1510 & $\begin{array}{l}15 \\
13\end{array}$ \\
\hline 9. & HLA-B-4402 & $\begin{array}{l}11 \\
10\end{array}$ & 9. & HLA-B-2705 & $\begin{array}{l}16 \\
15 \\
12\end{array}$ \\
\hline 10. & HLA-B-5101 & 10 & 10. & HLA-B-2709 & $\begin{array}{l}13 \\
12\end{array}$ \\
\hline \multirow[t]{3}{*}{11.} & RT1.A1 & $\begin{array}{c}12 \\
10 \\
9\end{array}$ & 11. & HLA-B-4402 & $\begin{array}{l}15 \\
14\end{array}$ \\
\hline & & & 12. & HLA-B-5101 & 13 \\
\hline & & & 13. & RT1.A1 & 8 \\
\hline
\end{tabular}

allows the use of multiple antigens with varying affinities and avidities to the HLA molecules in a patient. There is a lot of evidence suggesting that one of the mechanisms by which a tumor evades immune surveillance is by downregulating surface antigens [25] and hence univalent antigen target is not very effective. Using a polyvalent peptide vaccine could potentially bypass this phenomenon by making multiple epitopes visible to the immune system. A distinct advantage in having peptide based vaccines is their ability to be presented via MHC class II molecules leading to a long term CD4+ T cell and $\mathrm{B}$ cell response as well as to be cross presented via MHC Class I leading to a CD8+ CTL response.

Various attempts have been made at generating peptide vaccines against different cancers. AWT1 peptide based vaccine in advanced pancreatic cancer, 


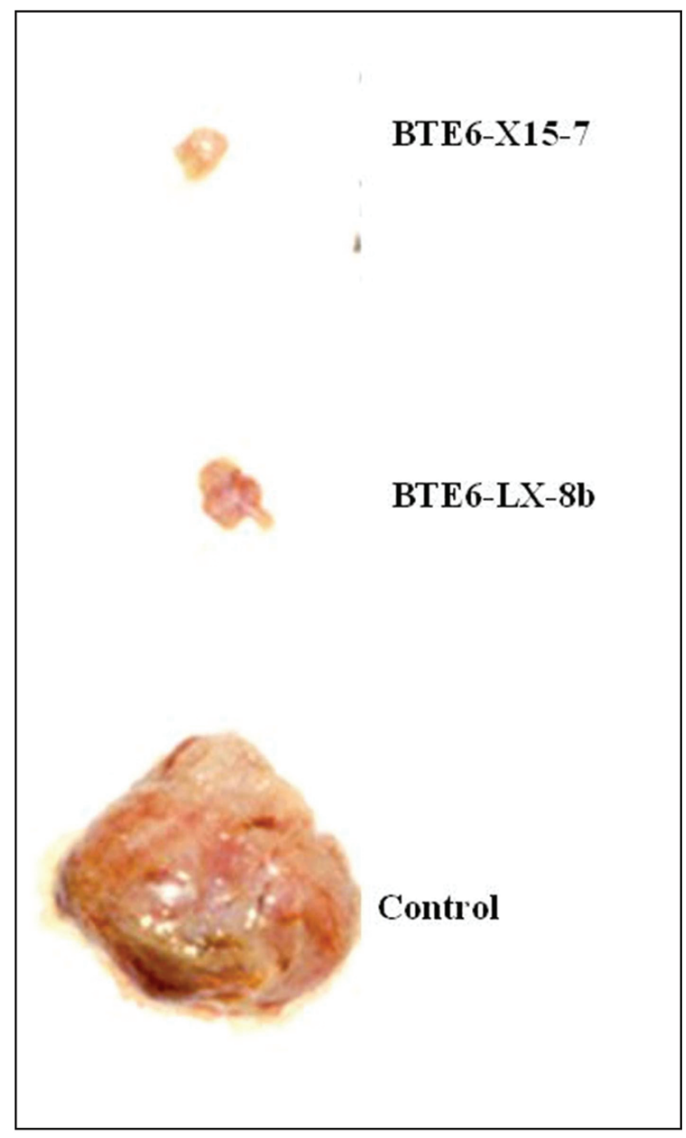

Figure 3 Effect of vaccination of synthetic peptides challenged by MAT-LyLu tumor cells: Hsp96 mimotopes, BTE6-LX-8b and BTE6-X15-7, vaccination show tumor protection. Vaccination with synthetic peptides abrogation of MAT-LyLu tumor [56].

when combined with gemcitabine showed a $29 \% 1$ year survival and a median survival of 8.1 months [26]. Another peptide based vaccine used E75 derived from the HER2 protein (nelipepimut-S) along with GMCSF as an adjuvant for treatment of breast cancer. This vaccine showed a high efficacy with almost $90 \%$ disease free survival compared with a control group [27]. A trial using a vaccine targeting a hTERT, Vx-001, in patients with advanced non-small cell lung carcinoma also showed promise with a TERT specific immunological response mounted; correlated to a better prognosis and higher progression free survival [28]. 
One patient completed the 2-year vaccine therapy.

- Two patients are in maintenance vaccine therapy.

- No significant toxicity

- Patients showed clinical responses

- Regression of tumor nodules in the lungs

- Survival beyond the expected survival time

- Patients showed the induction of anti-melanoma immunity .

- Delayed-type hypersensitivity response (DTH response) test,

- Lymphocyte proliferation assay

- Cytotoxic T-lymphocyte (CTL) assay

- Antibody assay.

Figure 4 Compassionate pilot clinical trial: A melanoma vaccine was generated using recombinant vaccinia virus expressing IL-2 and cell lysates prepared from 5 primary proprietary melanoma cell lines. Three patients were vaccinated with the melanoma vaccine (DC-MelVac) consisting of recombinant vaccinia virus and DC-lysate immunogen. Vaccination resulted in increased overall survival and regression of tumor nodules.

Table 3 Immune modulation of treated patients: Three patients were treated with the melanoma vaccine in a Phase I compassionate clinical trial. Anti-melanoma immune response was observed due to induction of delayed type hypersensitivity reactions (DTH), cytotoxic-T lymphocyte presence (CTL), lymphocyte proliferation (Pro) and antibody induction (Ab)

\begin{tabular}{|cccccc|}
\hline \multicolumn{2}{|c}{$\underline{\text { Patient }}$} & $\underline{\text { DTH }}$ & $\underline{\mathrm{CTL} / \mathrm{p}}$ & $\underline{\text { Pro. }}$ & $\underline{\mathrm{Ab}}$ \\
\hline \multirow{2}{*}{$\# 1$} & Pre & $2 \mathrm{~mm}$ & + & - & + \\
& Post & $5 \mathrm{~mm}$ & ++ & + & ++ \\
\multirow{2}{*}{$\# 2$} & Pre & $3 \mathrm{~mm}$ & + & - & + \\
& Post & $5 \mathrm{~mm}$ & + & - & ++ \\
\multirow{2}{*}{$\# 3$} & Pre & $3 \mathrm{~mm}$ & + & - & ++ \\
& Post & $12 \mathrm{~mm}$ & +++ & ++ & ++ \\
\hline
\end{tabular}

We are the first group to define a multivalent peptide mimotope based immunogen that fulfills the criteria laid down for a robust immune response. It is synthetic, can be easily manufactured and needs no refrigeration, stimulating both arms of the adaptive immune response. It can be adapted to any cancer, individualized, combined with any adjuvant and since used extraneously 
can easily be controlled for dose and frequency of administration making it a truly versatile immunogen. The use of vaccinia based multivalent tumor antigen retrieval system has been tried in a compassionate clinical trial with encouraging results. In this trial, we established the premise that dendritic cells pulsed with recombinant IL-2 gene encoded vaccinia virus infected melanoma oncolysates, derived from five primary melanoma cell lines, present the MAAs present in the oncolysate to the CTLs [29, 30]. This vaccine (DC-MelVac) has now been approved by the FDA for a phase 1 clinical trial. Initial results with prior generation of the vaccine showed delayed type hypersensitivity and a multivalent immune response [31].

Need for an Adjuvant Boost: Several studies have aimed to improve the efficacy of peptide based vaccines. The initial studies to improve efficacy started with the addition of an adjuvant alongwith the peptide vaccine. GMCSF was one of the adjuvants used in a few studies, which showed varying results. A study conducted in stage $3 \mathrm{c}$ melanoma patients indicated that GM-CSF administration alone led to an improvement in melanoma specific survival [32]. Another study, using GM-CSF alongwith a whole cell vaccine in melanoma patients, showed that GM-CSF was not a good adjuvant and infact had negative impacts [33]. A study carried out by Slingluff et al. [34] using 12 melanoma peptides, with and without GM-CSF as an adjuvant, also demonstrated the ineffectiveness of using GM-CSF as an adjuvant [35]. These varied results suggest the need for a tightly controlled study in order to determine causes for variation, effective doses and schedules. The premise of using IL-2 in our vaccinia based studies rests in the ability of the cytokine to recruit and activate $\mathrm{T}$ cells, mounting a stronger immune response to a specific peptide [36]. The side effects at high dose include flu like symptoms, fluid retention and organ dysfunction at high doses [37].

The Use of Targeted Therapy: In order to ensure delivery of vaccines to the lymph nodes, the site for coordination of immune responses, several groups have used tools that rest in the fields of materials sciences and tissue engineering. One group demonstrated that antigens covalently conjugated to virus sized inert nano particles were potent immunogens; mounting an immune response by localizing to the draining lymph nodes and initiating dendritic cell cross priming to CD8+ T cells [38]. Another group recently studied the effect of targeting immunosuppressed, antigen rich tumor draining lymph nodes in mice using a nanoparticulate cancer vaccine in a B16-F10 melanoma mouse model. They discovered that despite the immunosuppressive environment of the tumor draining lymph nodes, targeting the nano particle conjugated with tyrosinase (a TAA in melanoma) and a $\mathrm{CpG}$ adjuvant led 
to a more robust immune response compared to targeting the vaccine to non-tumor draining lymph nodes [39]. Another extremely interesting study involved coating polylactic-co-glycolic acid (PLGA) nanoparticles with cell membranes from B16-F10 melanoma cells. This study demonstrated a method to specifically target cancer antigens to DCs inducing maturation in vitro [40]. In addition to nanoparticles, some groups have exploited albumin due to its ability to traffic from the vasculature into the lymphatics. Conjugating peptide antigens and $\mathrm{CpG}$ as an adjuvant to saturated lipid tails leads to the peptide being docked into the fatty acid binding pockets of albumin. This albumin conjugate promoted higher homing into the lymph nodes compared to soluble antigens and adjuvants. It led to a 30 -fold increase in T cell priming, enhancing anti-tumor cytotoxicity while keeping systemic toxicity low [41]. All of these promising methods are able to deliver a positive stimulatory immune response; however they don't address the immunosuppressive environment of the tumor which leads to inactivation of T cells due to tumor induced secretory molecules as well as engagement of negative regulatory receptors and ligands.

The Use of Checkpoint Inhibitors: The discovery of diminished antitumor in vivo activity by effector T cells that are primed in vitro called for a new criteria for screening of cells during an adoptive $\mathrm{T}$ cell transfer therapy [42]. The immunosuppressive tumor microenvironment plays a critical role in rendering primed and active T cells inactive, enabling tumor evasion [6, 43]. Cytotoxic T Lymphocyte Antigen-4 (CTLA-4) is a cell surface receptor expressed on activated $\mathrm{T}$ cells. It binds to B7-1/2 on antigen presenting cells with a high affinity, inactivating $\mathrm{T}$ cells. This receptor plays a major role in regulation and maintenance of $\mathrm{T}$ cell homeostasis [44, 45]. Another $\mathrm{T}$ cell surface molecule, Programmed Cell Death-1 (PD-1) also plays an inhibitory role, inactivating $\mathrm{T}$ cells when engaged with its ligands; often expressed on tumor cells [46]. Exploitation of these two pathways, led to the development of two new FDA approved monoclonal antibodies, ipilimumab (anti CTLA-4) and nivolumab (anti PD-1) for use in metastatic melanoma by Bristol Squibb Meyers [47]. These antibodies in addition to pembrolizumab (anti PD-1) developed by Merck, and approved for PD-L1 expressing metastatic non-small cell lung cancer, have now become the talk of the town. With their ability to prevent $\mathrm{T}$ cell inactivation in the tumor microenvironment, these antibodies showed promising results in clinical trials for metastatic melanoma [48-50]. A list of possible checkpoint inhibitors that are clinically relevant are provided in Figure 5. Drawbacks of anti-CTLA4 therapy include a plethora of hard to manage side effects like Grave's Disease [51], pulmonary toxicities [52], 


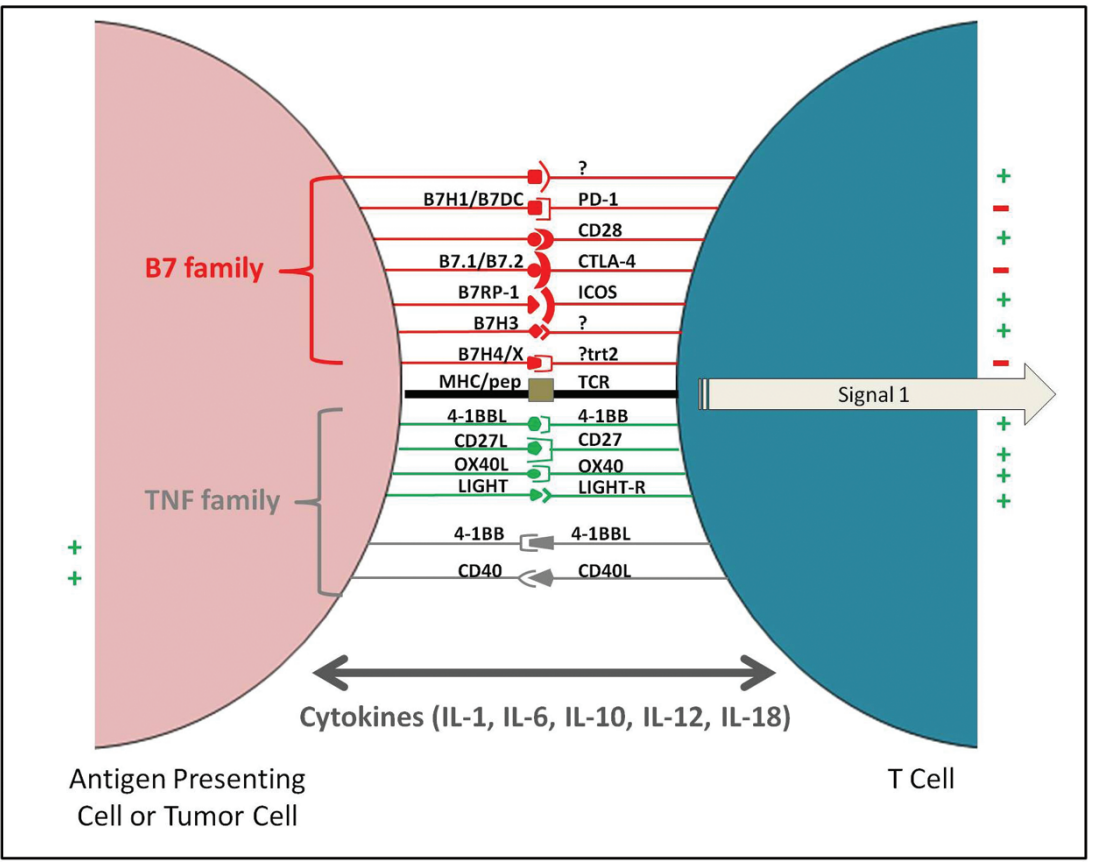

Figure 5 APC-T cell interactions utilizing B7 and TNF family of receptors and ligands that are the key elements of negative checkpoint inhibitors: This strategy utilizes the host immune response against cancer cells. Vaccination could lead to the presentation of tumor associated antigens by host APCs (antigen presenting cells) activating T cells. Aided by checkpoint inhibitors (e.g. anti-CTLA-4 antibody) the inactivation of the T cells would be curtailed, consequently potentiating the host immune response against cancer cells.

gastrointestinal toxicities [53] as well as immune related adverse events [54]. Combination therapies using multiple checkpoint inhibitors are now being tried in various cancers; the first one to be approved is a combination of nivolumab and ipilimumab in metastatic melanoma [47].

A Case for Combining Targeted Peptide Vaccines and Check-Point Inhibitors for Therapy: With the evolution of drugs preventing $\mathrm{T}$ cell inactivation and promoting effective $\mathrm{T}$ cell activation, we are now in the midst of a whole new paradigm in cancer immunotherapy. The low immunogenicity seen in case of certain peptide based vaccines can be attributed to the immunosuppressive environment in the tumor. Adding a checkpoint inhibitor to the peptide based vaccine therapies, could potentially lead to an enhanced immune response and effective tumor clearance. Furthermore, with the advent of targeted therapies, localizing peptide mimotope based vaccines or our 
vaccinia virus based oncolysate (DC-MelVac) to the tumor draining lymph nodes and coupling it with check-point inhibitors, would arm the immune system with a much needed one-two punch in order to combat tumors. This treatment regimen could lead to a prolonged antigen specific $\mathrm{T}$ cell priming and activation, and a higher overall survival and disease free progression in patients. The evolution of cancer immunotherapy and the data seen thus far encourages us to explore the option for this combinatorial therapy.

\section{Concluding Remarks}

We present the conundrum of the ongoing dilemma in cancer immunity that relates to the challenges of genomic instability based generation of immuneepitopes. We also present the novel patented technologies that allow us to generate multivalent vaccines that presumably can be tailor made to suit the modern need for individualized precision medicine and address the challenges of genomic instability mediated epitope generation. The $21^{\text {st }}$ century belongs to cancer eradication through the prudent combinatorial use of immunotherapy with other therapeutic modalities. To this end immunotherapy has be to fine tuned with respect to positive and negative stimulation and in this regard novel therapeutic regimens with our positive immune boosters need to be combined with checkpoint inhibitors. This fine tuning of these two arms in a natural environment of the immunocompetent host holds the promise of cancer eradication.

\section{Methods}

1. Single chain antibody ( $\mathbf{s c F v}$ ) library panning strategy: Antibody genes pertaining to $\mathrm{scFv}$ fragments with randomized CDR3 regions were inserted into a phagemid and fused with M13 bacteriophage coat/capsid protein. This enabled the formation of a large repertoire of an single chain antibody phage library displaying the variable CDR3 sequence on the phage surface. These phages are single stranded phagemids, since they possess both a viral and bacterial origin of replication, can replicate in E.coli providing a vast repertoire $\left(10^{8}-10^{9}\right)$ of single chain antibodies for screening processes. Hsp 96-peptide complex is panned against the single chain antibody ( $\mathrm{scFv}$ ) phage library to ascertain specific clones. After three rounds of panning, the specific phage clones showing affinity and specificity to hsp96-peptide complex were isolated and analyzed for reactivity through ELISA. 
2. Single chain peptide library screening: The phage display peptide library is incubated with the single chain antibody ( $\mathrm{scFv}$ ) against tumor hsp96-peptide complex (MAT-LyLu hsp96-peptide complex) in microtiter plates. The bound phages are eluted and amplified in E.coli cells to get an enriched phage subset. Few more successive rounds of panning, elution and amplification are done to enrich for specificity as well as affinity to the target - tumor hsp96-peptide complex. The phage clones with the maximum specificity to the target protein are selected and sequenced to find the conformation of the peptide displayed on each specific phage clone. One such peptide generated was X-15, mimotope of MAT-LyLu hsp96-peptide complex, whose immunogenicity was analyzed and tumor protective effects determined through further animal experiments [55].

3. Vaccination efficacy: Two synthetic peptides were obtained by panning with scFv E6 clone specific for hsp96-peptide complex. Here LX-8b is a 12-mer peptide acting as a control of MAT-LyLu hsp96-peptide complex mimic, and X-15 is the linear 15-mer mimotope of MAT-LyLu hsp96-peptide complex obtained. The sequence of both the peptides is shown in Table 2. In order to check the immunogenicity of the peptides, syngeneic Copenhagen rats were immunized subcutaneously with 100 $\mu \mathrm{g}$ peptide/rat on day 0 , and $50 \mu \mathrm{g}$ peptide/rat on days 14 and 21 as booster doses. 10,000 MAT-LyLu cells were injected on day 14 as a tumor cell challenge. The challenge resulted in palpable tumors by day 10 in the control untreated group. However, there was delay in latency, incidence and tumor growth in X-15 peptide vaccinated group similar to the positive control LX-8b group (Figure 3) [56].

4. DC-MelVac compassionate trial: We have established 5 primary proprietary patient derived melanoma cells. Cell lysates were prepared from these cell lines using a vaccinia virus expressing IL-2. This preparation was used in a Phase I compassionate clinical trial of melanoma patients. Patients' dendritic cells were loaded with the lysate and matured to present the specific immunogens. Vaccine, containing the recombinant vaccinia virus and DC-lysate immunogen, was administered to three patients and a clinical response observed (Figure 4). Decrease in tumor nodules in lungs and increased overall survival was observed. No significant toxicity was noted. An anti-melanoma immune response was assessed by stimulation of delayed type hypersensitivity, lymphocyte proliferation, cytotoxic T-lymphocyte presence and antibody induction (Table 3) [29-31]. 


\section{Abbreviations}

$\begin{array}{ll}\text { APC } & \text { Antigen presenting cell } \\ \text { CDR3 } & \text { Complementarity determining region } \\ \text { CpG } & \text { 5-C-phosphate-G-3' } \\ \text { CTL } & \text { Cytotoxic T lymphocyte } \\ \text { CTLA-4 } & \text { Cytotoxic T-lymphocyte-associated protein } 4 \\ \text { DC } & \text { Dendritic cells } \\ \text { FDA } & \text { Food and Drug Administration } \\ \text { GMCSF } & \text { Granulocyte-macrophage colony-stimulating factor } \\ \text { HER2 } & \text { Receptor tyrosine-protein kinase erbB-2 } \\ \text { HLA } & \text { Human Leukocyte Antigen } \\ \text { HSP } & \text { Heat shock proteins } \\ \text { hTERT } & \text { Human Telomerase Reverse Transcriptase } \\ \text { IL-2 } & \text { Interleukin-2 } \\ \text { MAA } & \text { Melanoma associated antigen } \\ \text { MAT-LyLu } & \text { A rat prostate tumor cell line } \\ \text { MHC } & \text { Major Histocompatibility Complex } \\ \text { PBS/T } & \text { Phosphate Buffered Saline/with Tween 20 } \\ \text { PD-1 } & \text { Programmed cell death protein 1 } \\ \text { PD-L1 } & \text { Programmed death-ligand 1 } \\ \text { scFV } & \text { Single-chain variable fragment } \\ \text { TAA } & \text { Tumor associated antigen } \\ \text { TRA } & \text { Tumor rejection antigen } \\ \text { TSA } & \text { Tumor specific antigen } \\ \text { WT1 } & \text { Wilms tumor protein }\end{array}$

\section{Acknowledgements}

We acknowledge the financial assistance of AVT and the National Cancer Institute RO1 grant 1RO1CA131946 (RKT). The data in this article was part presented at the International Conference on Translational Medicine: Emerging Trends in Biomedicine, Biotechnology and Stem Cell Research Present Status and Future Prospect, February 19-20, 2016 as a keynote speaker address.

\section{References}

[1] Baldwin, R. W. (1955). Immunity to methylcholanthrene-induced tumours in inbred rats following atrophy and regression of the implanted tumours. Br. J. Cancer 9, 652-657. 
[2] Foley, E. J. (1953). Antigenic properties of methylcholanthrene-induced tumors in mice of the strain of origin. Cancer Res. 13, 835-837.

[3] Prehn, R. T., and Main, J. M. (1957). Immunity to methylcholanthreneinduced sarcomas. J. Natl. Cancer Inst. 18, 769-778.

[4] Klein, G., Sjogren, H. O., Klein, E., and Hellstrom, K. E. (1960). Demonstration of resistance against methylcholanthrene-induced sarcomas. Cancer Res. 20, 1561-1572.

[5] Greenman, C., Stephens, P., Smith, R., Dalgliesh, G. L., et al. (2007). Patterns of somatic mutation in human cancer genomes. Nature 446, 153-158.

[6] Hanahan, D., and Weinberg, R. A. (2011). Hallmarks of cancer: the next generation. Cell 144, 646-674.

[7] Schumacher, T. N., and Schreiber, R. D. (2015). Neoantigens in cancer immunotherapy. Science 348, 69-74.

[8] Coulie, P. G., Lehmann, F., Lethé, B., Herman, J., et al. (1995). A mutated intron sequence codes for an antigenic peptide recognized by cytolytic T lymphocytes on a human melanoma. Proc. Natl. Acad. Sci. U.S.A. 92, 7976-7980.

[9] Baldwin, R. W. (1955). Immunity to transplanted tumour: the effect of tumour extracts on the growth of homologous tumours in rats. Br. J. Cancer 9, 646-651.

[10] Srivastava, P. (2002). Interaction of heat shock proteins with peptides and antigen presenting cells: chaperoning of the innate and adaptive immune responses. Annu. Rev. Immunol. 20, 395-425.

[11] Suto, R., and Srivastava, P. K. (1995). A mechanism for the specific immunogenicity of heat shock protein-chaperoned peptides. Science 269, $1585-1588$.

[12] Binder, R. J., Han, D. K., and Srivastava, P. K. (2000). CD91: a receptor for heat shock protein gp96. Nat. Immunol. 1, 151-155.

[13] Schlesinger, M. J. (1990). Heat Shock Proteins: minireview. J. Biol. Chem. 265, 12111-12114.

[14] Udono, H., and Srivastava, P. K. (1993). Heat shock protein 70-associated peptides elicit specific cancer immunity. J. Exp. Med. 178, 1391-1396.

[15] Calderwood, S. K., Stevenson, M. A., and Murshid, A. (2012). Heat shock proteins, autoimmunity, and cancer treatment. Autoimmune Dis. 2012:486069.

[16] Bunting, S. F., and Nussenzweig, A. (2013). End-joining, translocations and cancer. Nat. Rev. Cancer 13, 443-454. 
[17] Srivastava, P. K. (2015). Neoepitopes of cancers: looking back, looking ahead. Cancer Immunol. Res. 3, 969-977.

[18] Suriano, R., Rajoria, S., George, A. L., Geliebter, J., et al. (2013). Ex vivo derived primary melanoma cells: implications for immunotherapeutic vaccines. J. Cancer 4, 371-382.

[19] Suriano, R., Rajoria, S., George, A., Jussim, C., et al. (2012). Proceedings of the 103rd Annual Meeting of the American Association for Cancer Research: multivalent melanoma vaccine using primary human melanoma cells and a vaccinia virus based antigen retrieval system. Cancer Res. 72, 1569.

[20] Wallack, M. K., McNally, K. R., Leftheriotis, E., Seigler, H., et al. (1986). A Southeastern Cancer Study Group phase I/II trial with vaccinia melanoma oncolysates. Cancer 57, 649-655.

[21] Wallack, M. K., Meyer, M., Bourgoin, A., Doré, J. F., et al. (1983). A preliminary trial of vaccinia oncolysates in the treatment of recurrent melanoma with serologic responses to the treatment. J. Biol. Response Mod. 2, 586-596.

[22] George, A. L., Suriano, R., Rajoria, S., Osso, M. C., et al. (2015). PLX4032 mediated melanoma associated antigen potentiation in patient derived primary melanoma cells. J. Cancer 6, 1320-1330.

[23] Suriano, R., Tuli, N. Y., Geliebter, J., Tiwari, R. K., et al. (2015). Proceedings of the 106th Annual Meeting of the American Association for Cancer Research: novel targeted combinational therapies for melanoma. Cancer Res. 75, 3540.

[24] Larché, M. (2008). Determining MHC restriction of T-cell responses. Methods Mol. Med. 138, 57-72.

[25] Khanna, R. (1998). Tumour surveillance: missing peptides and MHC molecules. Immunol. Cell Biol. 76, 20-26.

[26] Nishida, S., Koido, S., Takeda, Y., Homma, S., Komita, H., Takahara, A., et al. (2014). Wilms tumor gene (WT1) peptide-based cancer vaccine combined with gemcitabine for patients with advanced pancreatic cancer. J. Immunother. 37, 105-114.

[27] Mittendorf, E. A., Clifton, G. T., Holmes, J. P., Schneble, E., et al. (2014). Final report of the phase I/II clinical trial of the E75 (nelipepimut-S) vaccine with booster inoculations to prevent disease recurrence in highrisk breast cancer patients. Ann. Oncol. 25, 1735-1742.

[28] Kotsakis, A., Papadimitraki, E., Vetsika, E. K., Aggouraki, D., et al. (2014). A phase II trial evaluating the clinical and immunologic response 
of HLA-A2(+) non-small cell lung cancer patients vaccinated with an hTERT cryptic peptide. Lung Cancer 86, 59-66.

[29] Jack, A. M., Aydin, N., Montenegro, G., Alam, K., et al. (2007). A novel dendritic cell-based cancer vaccine produces promising results in a syngenic CC-36 murine colon adenocarcinoma model. J. Surg. Res. 139, 164-169.

[30] Aydin, N., Jack, A., Montenegro, G., Boyes, C., et al. (2006). Expression of melanoma-associated antigens in human dendritic cells pulsed with an interleukin-2 gene encoded vaccinia melanoma oncolysate (rIL-2VMO). Cancer Biol. Ther. 5, 1654-1657.

[31] Suriano, R., Rajoria, S., George, A. L., Geliebter, J., et al. (2013). Followup analysis of a randomized phase III immunotherapeutic clinical trial on melanoma. Mol. Clin. Oncol. 1, 466-472.

[32] Grotz, T. E., Kottschade, L., Pavey, E. S., Markovic, S. N., et al. (2014). Adjuvant GM-CSF improves survival in high-risk stage iiic melanoma: a single-center Study. Am. J. Clin. Oncol. 37, 467-472.

[33] Faries, M. B., Hsueh, E. C., Ye, X., Hoban, M., et al. (2009). Effect of granulocyte/macrophage colony-stimulating factor on vaccination with an allogeneic whole-cell melanoma vaccine. Clin. Cancer Res. 15, 7029-7035.

[34] Slingluff, C. L., Petroni, G. R., Yamshchikov, G. V., Hibbitts, S., et al. (2004). Immunologic and clinical outcomes of vaccination with a multiepitope melanoma peptide vaccine plus low-dose interleukin-2 administered either concurrently or on a delayed schedule. J. Clin. Oncol. 22, 4474-4485.

[35] Slingluff, C. L., Petroni, G. R., Olson, W. C., Smolkin, M. E., et al. (2009). Effect of granulocyte/macrophage colony-stimulating factor on circulating CD8+ and CD4+ T-cell responses to a multipeptide melanoma vaccine: outcome of a multicenter randomized trial. Clin. Cancer Res. $15,7036-7044$.

[36] Overwijk, W. W., Theoret, M. R., and Restifo, N. P. (2000). The future of interleukin-2: enhancing therapeutic anticancer vaccines. Cancer J. Sci. Am. 6(Suppl. 1), S76-S80.

[37] Rosenberg, S. A., Lotze, M. T., Yang, J. C., Aebersold, P. M., et al. (1989). Experience with the use of high-dose interleukin-2 in the treatment of 652 cancer patients. Ann. Surg. 210, 474-485.

[38] Fifis, T., Gamvrellis, A., Crimeen-Irwin, B., Pietersz, G. A., et al. (2004). Size-dependent immunogenicity: therapeutic and protective properties of nano-vaccines against tumors. J. Immunol. 173, 3148-3154. 
[39] Jeanbart, L., Ballester, M., de Titta, A., Corthésy, P., et al. (2014). Enhancing efficacy of anticancer vaccines by targeted delivery to tumor-draining lymph nodes. Cancer Immunol. Res. 2, 436-447.

[40] Fang, R. H., Hu, C. M., Luk, B. T., Gao, W., et al. (2014). Cancer cell membrane-coated nanoparticles for anticancer vaccination and drug delivery. Nano Lett. 14, 2181-2188.

[41] Liu, H., Moynihan, K. D., Zheng, Y., Szeto, G. L., et al. (2014). Structurebased programming of lymph-node targeting in molecular vaccines. Nature 507, 519-522.

[42] Gattinoni, L., Klebanoff, C. A., Palmer, D. C., Wrzesinski, C., et al. (2005). Acquisition of full effector function in vitro paradoxically impairs the in vivo antitumor efficacy of adoptively transferred CD8+ T cells. J. Clin. Invest. 115, 1616-1626.

[43] Gajewski, T. F., Schreiber, H., and Fu, Y.-X. (2013). Innate and adaptive immune cells in the tumor microenvironment. Nat. Immunol. 14, 1014-1022.

[44] Walunas, T. L., Bakker, C. Y., and Bluestone, J. A. (1996). CTLA-4 ligation blocks CD28-dependent T cell activation. J. Exp. Med. 183, 2541-2550.

[45] McCoy, K. D., and Le Gros, G. (1999). The role of CTLA-4 in the regulation of T cell immune responses. Immunol. Cell Biol. 77, 1-10.

[46] Jin, H.-T., Ahmed, R., and Okazaki, T. (2011). Role of PD-1 in regulating T-cell immunity. Curr. Top. Microbiol. Immunol. 350, 17-37.

[47] Wolchok, J. D., Kluger, H., Callahan, M. K., Postow, M. A., et al. (2013). Nivolumab plus ipilimumab in advanced melanoma. N. Engl. J. Med. $369,122-133$.

[48] Hoos, A., Ibrahim, R., Korman, A., Abdallah, K., et al. (2010). Development of ipilimumab: contribution to a new paradigm for cancer immunotherapy. Semin. Oncol. 37, 533-546.

[49] Hodi, F. S., O’Day, S. J., McDermott, D. F., Weber, R. W., et al. (2010). Improved survival with ipilimumab in patients with metastatic melanoma. N. Engl. J. Med. 363, 711-723.

[50] Brahmer, J. R., Drake, C. G., Wollner, I., Powderly, J. D., et al. (2010). Phase I study of single-agent anti-programmed death-1 (MDX-1106) in refractory solid tumors: safety, clinical activity, pharmacodynamics, and immunologic correlates. J. Clin. Oncol. 28, 3167-3175.

[51] Azmat, U., Liebner, D., Joehlin-Price, A., Agrawal, A., et al. (2016). Treatment of ipilimumab induced graves' disease in a patient with 
metastatic melanoma. Case Rep. Endocrinol. 2016, 2087525. doi: $10.1155 / 2016 / 2087525$.

[52] Mailleux, M., Cornélis, F., Colin, G., and Baurain, J. F. (2015). Unusual pulmonary toxicity of ipilimumab treated by macrolides. Acta Clin. Belg. doi: 10.1179/2295333715Y.0000000047 [Epub ahead of print].

[53] Verschuren, E. C., van den Eertwegh, A. J., Wonders, J., Slangen, R. M., et al. (2015). Clinical, endoscopic, and histologic characteristics of ipilimumab-associated colitis. Clin. Gastroenterol. Hepatol. doi: 10.1016/j.cgh.2015.12.028 [Epub ahead of print].

[54] Horvat, T. Z., Adel, N. G., Dang, T. O., Momtaz, P., et al. (2015). Immunerelated adverse events, need for systemic immunosuppression, and effects on survival and time to treatment failure in patients with melanoma treated with ipilimumab at Memorial Sloan Kettering Cancer Center. J. Clin. Oncol. 33, 3193-3198.

[55] Chaudhuri, D., Suriano, R., Mittelman, A., and Tiwari, R. K. (2009). Targeting the immune system in cancer. Curr. Pharm. Biotechnol. 10, 166-184.

[56] Shanmugam, A., Suriano, R., Goswami, N., Chaudhuri, D., et al. (2011). Identification of peptide mimotopes of gp96 using single-chain antibody library. Cell Stress Chaperones 16, 225-234.

[57] Shanmugam, A., Suriano, R., Chaudhuri, D., Rajoria, S., et al. (2011). Identification of PSA peptide mimotopes using phage display peptide library. Peptides 32, 1097-1102.

\section{Biographies}

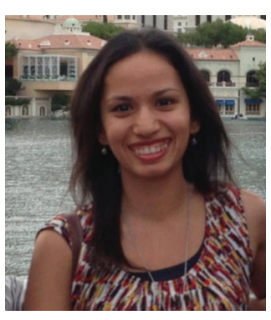

N. Tuli is a Ph.D. candidate under the mentorship of Dr. Raj K. Tiwari at New York Medical College. Her curiosity in the medical sciences led her to enter medical school at Government Medical College (GMC, Surat, Gujarat, India) very early on in her career. After getting her M.B.B.S., Doctor of Medicine, in 2009, she practiced as a general physician and resident medical 


\section{$54 \quad$ N. Tuli et al.}

officer in private as well as government hospitals for 2 years. While working on a diphtheria project, her interest piqued towards the field of basic sciences and translational research. Subsequently, Dr. Tuli got her Master of Science in Pharmacology from New York Medical College in 2015. Her master research review was focused on metastatic castrate resistant prostate cancer. While pursuing her master's, she worked as a research associate in cancer immunology with focus on thyroid cancer, melanoma and breast cancer. Currently, she is continuing her research aspirations as a Ph.D. candidate in Dr. Tiwari's laboratory in Department of Microbiology and Immunology, New York Medical College. Her project focuses on thyroid tumor microenvironment - the mutual cooperation between inflammatory cells (including their secretome) and thyroid cancer epithelial cells to promote metastatic phenotype.

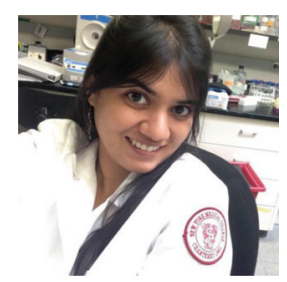

R. Maniyar is currently a graduate student in Dr. Tiwari's laboratory at New York Medical College with a research focus in melanoma therapeutics. She completed her Bachelor's degree from University of Mumbai, India in 2008 with a major in Life Sciences. Her interest in immunology propelled her to pursue her Master's degree in Immunology from King's College London, United Kingdom in 2009. Her master's thesis focused on developing an antibody-antigen-adjuvant conjugate based dendritic cell vaccine for cancer immunotherapy. Following this, she worked in industry in India for five years in the field of regenerative medicine, focusing on product discovery and development in stem cells and growth factor concentrates. In an effort to further satiate her curiosity and desire to explore cancer immunotherapy, she entered the Ph.D. program in the Department of Microbiology and Immunology. Her current research focus centers on checkpoint inhibitors in melanoma therapy, their significance and possible ways to enhance their efficacies. 


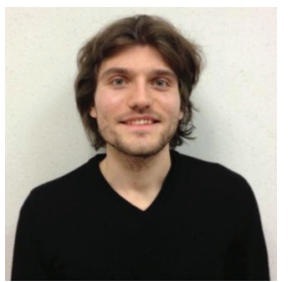

R. Bednarczyk attended Loyola University Chicago as an undergraduate and earned a Bachelor of Science in Biology with Molecular Emphasis in 2009. Following his undergraduate studies, he volunteered at Advocate Christ Medical Center in Oak Lawn, Illinois from 2009-2010. Subsequently, he entered New York Medical College for graduate studies and earned a Master of Science in Microbiology and Immunology in 2012. His master's thesis focused on Toxoplasma gondii, and its resistance to the innate immune response. To further pursue his research interests, he entered the Ph.D. program in the Department of Microbiology and Immunology program in the fall of 2012. His current research focuses on the importance of chronic inflammation as a major mediator of breast cancer development and progression. Specifically, focusing on the roles of pro-inflammatory tumor-associated macrophages (TAMs) including TAMs secretory factors - cytokines and exosomes, in breast cancer progression. During this time, he also worked as an adjunct instructor for Genetics Laboratory at Lander College for Men in the fall of 2015.

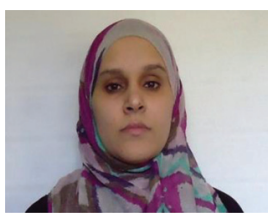

G. Ben Rahoma graduated from the School of Medicine, Seventh of April University, Az-Zawiyah, Libya (August, 2006) with M.B.B.S, Doctor of Medicine. After graduation, she was employed as a general physician at Second of March Polyclinic, Az-Zawiyah, Libya (Oct, 2006-June, 2008). At the same time, she was working as a teaching assistant in the Department of Microbiology, School of Medicine, Seventh of April University, (2006-2008). Her passion for science coupled with hard work was awarded a Scholarship for Outstanding Teaching Assistant. In 2010, she started her Master's program in the Department of Microbiology \& Immunology, New York Medical College. Her master's thesis involved development and application of a 
simple molecular rapid RT-PCR for detection and differentiation of Influenza A viruses. After finishing her master's in May 2013, she was admitted to the Ph.D. program, Microbiology and Immunology Department, New York Medical College. Currently, she is working under the mentorship of Dr. Raj K. Tiwari, on using endothelial progenitor cell exosomes as a delivery platform for targeting tumor angiogenesis using antiestrogens.

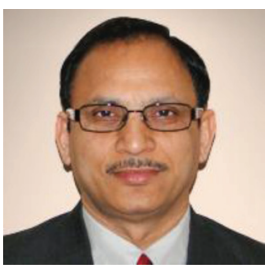

S. Singh earned his Ph.D. in Horiculture-Botany from the Banaras Hindu University in 1993 after finishing BSc (1985) and M.Sc (1988) from the same university. After completion of his doctorate, he started working as a junior research fellow in the Department of Botany, Banaras Hindu University. He quickly climbed the ladder to the position of Research Associate, having completed various projects sponsored by the Ministry of Environment and Forests as well as the Ministry of Coal, Govt. of India. Consequently, Dr. Singh bagged tenure position as a Senior Scientist and Head of the institution in Virendra Kumar Singh Krishi Vigyan Kendra, Unnao, Uttar Pradesh, India. For more than a decade, he worked as a material handler for 3M Canada Corporation in the healthcare division (2004-2016). Currently, he is a research associate coordinator at New York Medical College overlooking the clinical translational research projects.

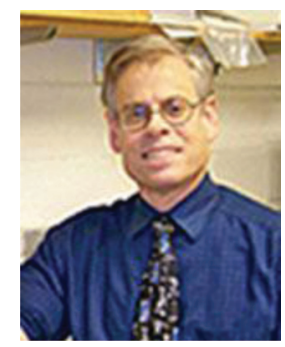

J. Geliebter is a professor in the Department of Microbiology and Immunology and Otolaryngology at New York Medical College. He received his 
undergraduate degree from Hebrew University of Jerusalem, Israel in Biology and his Doctorate from State University of New York Health Science Center at Brooklyn. His current research focus is papillary thyroid cancer, its molecular pathogenesis, and the effects of androgens on its progression and etiology. At New York Medical College Dr. Geliebter also holds the positions of Regional Vice President of the Executive Committee of Faculty Senate as well as the Course Director of the Medical Microbiology program. Loved by students and faculty alike, Dr. Geliebter has received numerous awards for this teaching and dedication as a mentor.

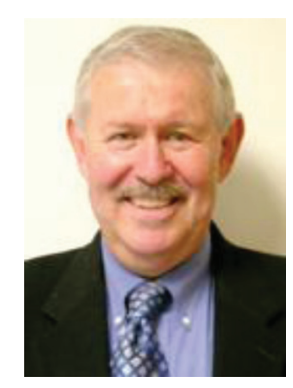

A. Mittelman is a practicing oncologist and an Associate Professor in the Department of Medicine, New York Medical College. He received his MD from Autonomous University of Guadalajara in 1977. He completed his internship and residency from Downstate-Kings County Medical Center, Brooklyn, New York (1981). Subsequently he achieved fellowship in Oncology, rising to the ranks of Adjunct Attending in Development Chemotherapy, Memorial Sloan Kettering Cancer Center, New York, New York (1987). His research interest to treat cancers brought him to New York Medical College/Westchester County Medical Center, Valhalla, New York, quickly advancing from an assistant attending to an associate professor position. He is also cofounder and CEO of AV therapeutics whose mission is to bring significant immunobiologicals to the clinic. 


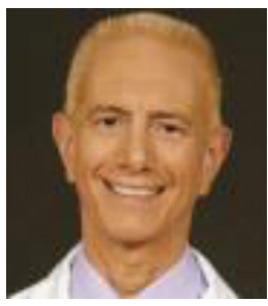

M. Wallack is the Chief of Surgery at Metropolitan Hospital, New York. He also holds the position of Vice-Chair of the Department of Surgery at New York Medical College. He received his MD from the University of Pittsburgh School of Medicine and completed his residency in general surgery from The University of Pennsylvania Health System. He has had an active practice for over 30 years. Dr. Wallack was instrumental in developing and clinically testing an FDA approved vaccinia virus based melanoma vaccine, and holds a patent for the same. His passion for research and improving patient health care is second to none.

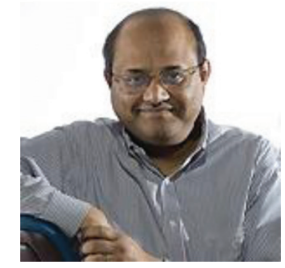

D. Banerjee is an Associate Dean - Global Initiative, at Graduate School of Biomedical Sciences, Rutgers University. Professor Banerjee has contributed greatly towards studies in structural biology, stem cells, cancer and gene therapy with over a hundred scientific publications to his credit. He obtained Ph.D. in Biochemistry from Calcutta University. Subsequently, he completed his postdoctoral fellowship training in Biology from McGill University, Canada (1983-1987). He worked as a Senior Scientist for Research on Hematology and Blood Transfusion in Calcutta (1987-1989). He also held the position of Research Scientist, Molecular Pharmacology Laboratory at the Sloan-Kettering Institute of New York (1989-1992). His insightful vision and immense intellectual ability brought him to Rutgers University, New Jersey as an Associate Professor of Medicine and Pharmacology at Robert Wood Johnson Medical School. He is the author of five patents awarded for his ground breaking research. 


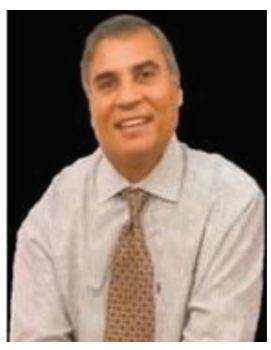

R. K. Tiwari is a Professor of Microbiology and Immunology, and Professor of Otolaryngology at New York Medical College. He attained a Master of Science in Biochemistry (1979) and earned a Doctor of Philosophy in Biochemistry (1983) from Calcutta University, India. He held the position of an Assistant Attending Biochemist in the Sloan-Kettering Institute for Cancer Research (1989-1991) and consequently was appointed as an Assistant Professor of Biochemistry in Department of Surgery at Cornell University Medical College, New York (1991-1996). Dr. Tiwari has mentored numerous undergraduate and graduate (M.S. and Ph.D.) students. He has been a Graduate Program Director for the Department of Microbiology and Immunology for over 15 years, always looking out for the students' best interest and providing them with the opportunity to acquire analytical skills for a career in academia, industry or translational research. Dr. Tiwari is a leader in cancer prevention and control with over 25 years of experience in cancer biology. His current research focuses on understanding the role of inflammation in breast and thyroid cancer specifically mitigated by infiltrating macrophages, as well as checkpoint inhibitors in melanoma therapy. In addition, Dr. Raj K. Tiwari is currently the Chief Scientific Officer of AV Therapeutics (2013-present) and is inventor of several patents involving Capridine and Cancer Vaccine. 
